Forum 2021 · 36:178

https://doi.org/10.1007/s12312-021-00911-6

Online publiziert: 26. Februar 2021

(c) Springer Medizin Verlag GmbH, ein Teil von Springer Nature 2021

\section{Krebsgesellschaft} Nordrhein-Westfalen e.V.

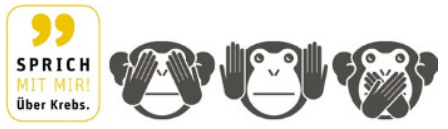

Krebsgesellschaft Nordrhein-Westfalen e.V.

Düsseldorf, Deutschland

\title{
Krebs macht vor Corona nicht halt! Krebsgesellschaft NRW startet Jugend-Contest mit Videos gegen Angst und Schweigen
}

Freunde, die Kollegen - mit einbezieht. Eine Krebserkrankung hat oftmals weitreichende Folgen auf das soziale Miteinander.

Trotz der Ernsthaftigkeit des Themas spricht der Wettbewerb bewusst junge Menschen an. „Die Unbefangenheit und Kreativität mit der sich junge Menschen auf den Umgang mit Krebs einlassen, sind wichtige gesellschaftliche Schritte hin zu mehr Offenheit und einem neuen Sprechen über Krebs, die weit über die Aktion hinauswirken", so Sandra Bothur. Die angstfreie Auseinandersetzung und der Mut zur Veränderung sind zentrale Themen des Video-Contests.

Projektpartner des Jugendvideo-Contests sind die AOK Rheinland/Hamburg sowie die Fachgruppe Selbsthilfe in der acht große NRW-Selbsthilfeverbände organisiert sind.

\section{Finale Video-Challenge}

Einsendeschluss für die Videos ist der 20. April 2021. Eine Jury - bestehend aus Initiatoren und Unterstützern des Wettbewerbs - sichtet alle Beiträge und nominiert die zehn besten Beiträge für die finale Video-Challenge. Das Gewinnervideo wird dann vor den Sommerferien via Live-Juryentscheid im Rahmen einer Präsenz- oder einer Online-Veranstaltung gekürt.

\section{Sprich mit mir! Über Krebs}

Der Jugendvideo-Contest ist Teil der Kampagne „Sprich mit mir! Über Krebs.“ mit der die Krebsgesellschaft NRW gesellschaftliche Tabus und Sprachlosigkeit bei Krebs in den Mittelpunkt öffentlicher Diskussion rückt. Inhaltlich knüpft der Wett- bewerb an die Schul-Video-Challenge an, die Anfang 2020 erfolgreich und noch als Präsenzveranstaltung durchgeführt werden konnte. Alle Beiträge der Kampagne sind auf dem YouTube-Kanal der Krebsgesellschaft NRW zu sehen.

Langfristiges Ziel der Kampagne ist die schrittweise Veränderung der sozialen Interaktion beim Thema Krebs zu mehr Offenheit, um sowohl Krebsbetroffene und Angehörige zu entlasten, als auch Außenstehenden Berührungsängste zu nehmen.

Weitere Informationen unter:

- www.sprichmitmirueberkrebs.de

- https://www.facebook.com/Krebsge-

sellschaft.NordrheinWestfalen

\section{Korrespondenzadresse}

\section{Dinah Oelschläger}

Krebsgesellschaft Nordrhein-Westfalen e.V.

Volmerswerther Straße 20,

40221 Düsseldorf, Deutschland

oelschlaeger@krebsgesellschaft-nrw.de

\section{Infobox Kampagnen-Elemente}

- Buch Sprich mit mir! Über Krebs. (2018-2021)

- Videoaktion in Kooperation mit der Fachgruppe Selbsthilfe (2018)

- Gesprächstrainings für Betroffene und Angehörige (2018-2019)

- Poetry Slam „Klartext statt Kopfkino“ (2019)

- Schulwettbewerb und Video-Challenge "Brich das Tabu" (2020)

- Social-Media-Aktionen und Bloggerkooperation (2019-2021)

- Jugendvideo-Contest "Nicht wegsehen. Weghören. Schweigen" (2021) 